Quim. Nova, Vol. 36, No. 9, 1332-1337, 2013

\title{
MODIFICAÇÃo E CARACTERIZAÇÃo DO RESÍDUO PÓ DE ACIARIA ELÉTRICA (PAE) PARA APLICAÇÃo EM REAÇÕES DE REDUÇÃO DE CROMO (VI)
}

\author{
Leandro Neves de Paula*, Luana Ap. dos Reis Giusto, Rodolfo Cesar Rodrigues Filho, Leandro Rossi Castilho e Fabiano \\ Magalhães ${ }^{\#}$ \\ Instituto de Química, Universidade Federal de Alfenas, Rua Gabriel Monteiro da Silva, 700, Centro, 37130-000 Alfenas - MG, Brasil \\ Marcelo Gonçalves Rosmaninho \\ Departamento de Química, ICEx, Universidade Federal de Minas Gerais, 31270-901 Belo Horizonte - MG, Brasil \\ Diana Quintão Lima \\ Departamento de Química, ICEx, Universidade Federal de Minas Gerais, 31270-901 Belo Horizonte - MG, Brasil
}

Recebido em 20/2/13; aceito em 18/7/13; publicado na web em 21/8/13

\begin{abstract}
MODIFICATION AND CHARACTERIZATION OF RESIDUE ELECTRIC ARC FURNACE DUST (EAFD) FOR APPLICATION IN CHROMIUM (VI) REDUCTION REACTIONS. In this study, electric arc furnace dust (EAFD) was thermally modified at different temperatures under $\mathrm{H}_{2}$ flow or charcoal in order to obtain reduced iron phases $\left(\mathrm{Fe}_{3} \mathrm{O}_{4}, \mathrm{FeO}\right.$ and $\left.\mathrm{Fe}^{0}\right)$. The formation of these phases was confirmed by powder X-ray diffraction. The tests performed for reducing $\mathrm{Cr}$ (VI) using resultant materials obtained after thermal treatment of the EAFD showed excellent results, with PAE600H (EAFD reduced at $600{ }^{\circ} \mathrm{C}$ under $\mathrm{H}_{2}$ flow) decreasing around $100 \%$ of the $\mathrm{Cr}(\mathrm{VI})$ in only 10 minutes of reaction. These results indicate the possibility of adding value to the residue, obtaining materials that offer great potential for environmental applications.
\end{abstract}

Keywords: electric arc furnace dust; hexavalent chromium; reduction.

\section{INTRODUÇÃO}

O pó de aciaria elétrica (PAE) é um resíduo rico em ferro que é gerado durante a produção do aço em siderúrgicas que utilizam o forno elétrico a arco (FEA). ${ }^{1}$ De acordo com Souza et al. (2010), para cada tonelada de aço produzida em aciarias com FEA são gerados cerca de 15 a $20 \mathrm{~kg}$ de PAE. ${ }^{1,2}$ Considerando que a produção de aço nacional em 2010 foi de aproximadamente 35,1 milhões de toneladas, ${ }^{3}$ pode-se estimar uma geração de ca. 614,2 mil toneladas de PAE. Por ser considerado um resíduo perigoso (contém $\mathrm{Pb}, \mathrm{Cd}, \mathrm{Cr}, \mathrm{Ni}, \mathrm{Fe}, \mathrm{Zn}$, etc), ${ }^{2,4-7}$ eles geram um alto custo para a indústria siderúrgica. Atualmente, cerca de $70 \%$ do PAE é disposto em aterros industriais, devido à falta de tecnologias alternativas de baixo custo para sua reciclagem. ${ }^{8} \mathrm{Uma}$ alternativa que tem despertado interesse nos últimos anos é o processo de estabilização química, ou vitrificação, que consiste na produção de materiais cerâmicos para construção civil utilizando como matéria prima o pó de aciaria elétrica, ${ }^{2,8-11}$ e em reações Fenton heterogêneo para degradação de contaminantes orgânicos. ${ }^{12}$

Devido ao alto teor de ferro no PAE, este pode ser um material interessante para aplicações em reações para remediação ambiental, como redução do $\mathrm{Cr}$ (VI) para $\mathrm{Cr}$ (III). As espécies $\mathrm{Fe}^{3+}$ e $\mathrm{Fe}^{2+}$ (como $\mathrm{Fe}_{2} \mathrm{O}_{3}$ e $\mathrm{Fe}_{3} \mathrm{O}_{4}$ ) presentes no $\mathrm{PAE}$ podem ser reduzidas à $\mathrm{Fe}^{0}$ a partir do tratamento térmico na presença de espécies redutivas como $\mathrm{H}_{2} \mathrm{e}$ carbono (Equações 1 a 3).

$$
\begin{gathered}
3 \mathrm{Fe}_{2} \mathrm{O}_{3}+\mathrm{H}_{2} / \mathrm{C} \rightarrow 2 \mathrm{Fe}_{3} \mathrm{O}_{4}+\mathrm{H}_{2} \mathrm{O} / \mathrm{CO}_{x} \\
\mathrm{Fe}_{3} \mathrm{O}_{4}+\mathrm{H}_{2} / \mathrm{C} \rightarrow 3 \mathrm{FeO}+\mathrm{H}_{2} \mathrm{O} / \mathrm{CO}_{x} \\
\mathrm{FeO}+\mathrm{H}_{2} / \mathrm{C} \rightarrow \mathrm{Fe}^{0}+\mathrm{H}_{2} \mathrm{O} / \mathrm{CO}_{\mathrm{x}}
\end{gathered}
$$

O ferro metálico formado pode, então, ser utilizado para reduzir o cromo hexavalente presente em efluentes contaminados (Equação 4).

*e-mail: leandroneves01@hotmail.com

"Endereço atual: Departamento de Química, Universidade Federal de Lavras, CP 3037, 37200-000 Lavras - MG, Brasil

$$
\mathrm{Fe}^{0}+\mathrm{HCrO}_{4}^{-}+7 \mathrm{H}^{+} \rightarrow \mathrm{Fe}^{3+}+\mathrm{Cr}^{3+}+4 \mathrm{H}_{2} \mathrm{O}
$$

A redução do cromo hexavalente utilizando materiais à base de ferro tem sido relatada na literatura como alternativa viável para descontaminação de efluentes e corpos d'água. Lago et al. (2010) utilizaram lama vermelha, ${ }^{13}$ compósitos de carvão/óxidos de ferro ${ }^{14,15}$ e resíduos industriais ${ }^{16,17}$ como agentes redutores do $\mathrm{Cr}(\mathrm{VI})$ presente em solução aquosa. O ferro metálico também tem sido estudado para reações de redução de contaminantes orgânicos como nitroaromáticos, ${ }^{18-24}$ organoclorados, ${ }^{25,26} \operatorname{corantes}^{15-17,27-29} \mathrm{e}$ pesticidas. $^{30-32}$

Sendo assim, neste trabalho as espécies $\mathrm{Fe}^{3+}$ e $\mathrm{Fe}^{2+}$ presentes no resíduo PAE foram reduzidas a $\mathrm{Fe}^{0}, \mathrm{FeO}$ e $\mathrm{Fe}_{3} \mathrm{O}_{4}$ e utilizadas em reações para conversão do $\mathrm{Cr}(\mathrm{VI})$ a $\mathrm{Cr}(\mathrm{III})$. Para tanto o PAE foi tratado termicamente utilizando-se $\mathrm{H}_{2}$ ou carvão vegetal, como agentes redutores, sob diferentes temperaturas $\left(400,600\right.$ e $\left.800{ }^{\circ} \mathrm{C}\right)$.

\section{PARTE EXPERIMENTAL}

\section{Obtenção dos compósitos}

A modificação química no pó de aciaria elétrica foi realizada através do tratamento térmico com o objetivo de reduzir as espécies $\mathrm{Fe}^{3+}$ e $\mathrm{Fe}^{2+}$ para obtenção das seguintes fases $\mathrm{Fe}_{3} \mathrm{O}_{4}, \mathrm{FeO}$ e $\mathrm{Fe}^{0} . \mathrm{O}$ tratamento térmico foi realizado utilizando um forno tubular em diferentes temperaturas $\left(400,600\right.$ e $\left.800{ }^{\circ} \mathrm{C}\right)$ de duas formas distintas. Na primeira, o PAE foi tratado termicamente sob fluxo de gás hidrogênio $\left(100 \mathrm{~mL} \mathrm{~min}^{-1}\right)$ durante 60 minutos. Neste procedimento foram obtidos os materiais PAE400H, PAE600H e PAE800H, conforme temperatura do tratamento. No segundo procedimento, o PAE foi misturado com carvão vegetal em uma proporção de 1:2 (m/m) e posteriormente aquecido sob fluxo de nitrogênio $\left(100 \mathrm{~mL} \mathrm{~min}{ }^{-1}\right)$ durante 60 minutos.

Posteriormente os materiais obtidos foram lavados com água destilada, separados magneticamente do carvão remanescente e secos 
a $60{ }^{\circ} \mathrm{C}$ durante ca. 4 horas em estufa. Neste procedimento foram obtidos os seguintes materiais: PAE400C, PAE600C e PAE800C. Após tratamento térmico todos os materiais foram armazenados em atmosfera inerte de $\mathrm{N}_{2}$.

O resíduo PAE utilizado neste trabalho foi fornecido por siderúrgicas situadas no estado de Minas Gerais.

\section{Caracterização}

Os materiais foram caracterizados por redução à temperatura programada (TPR) utilizando o equipamento Chembet 3000 Quantchrome com ca. $20 \mathrm{mg}$ de amostra aquecida a uma razão de $10{ }^{\circ} \mathrm{C} \mathrm{min}{ }^{-1}$ sob fluxo de $\mathrm{H}_{2}\left(20 \mathrm{~mL} \mathrm{~min}^{-1}\right)$. A difração de Raio-X foi realizada no equipamento Rigaku, utilizando radiação de $\mathrm{Cu} \mathrm{Ka}$, no intervalo entre 10 e $80^{\circ}$ a $4^{\circ} \mathrm{min}^{-1}$. Os espectros Mössbauer foram obtidos à temperatura ambiente em um espectrofotômetro modelo MA250 com fonte de ${ }^{57} \mathrm{Co}$ em matriz de Rh, utilizando $\alpha \mathrm{Fe}$ como referência. As análises de microscopia eletrônica de varredura (MEV) e as análises de espectroscopia de energia dispersiva (EDS) foram realizadas no Centro de Microscopia da UFMG em um equipamento Jeol JSM - 6360LV. A análise química foi realizada conforme Brehm et al. (2002) ${ }^{33}$ utilizando um espectrômetro de absorção atômica com chama (EAA-chama) Shimadzu modelo AA-7000.

\section{Redução de cromo (VI)}

Os testes de redução do $\mathrm{Cr}$ (VI) em solução aquosa foram realizados utilizando-se $5,00 \mathrm{~mL}$ de solução $50 \mathrm{mg} \mathrm{L}^{-1}$ de $\mathrm{Cr}$ (VI) e $60 \mathrm{mg}$ de PAE ou dos materiais obtidos. A cinética destas reações foi acompanhada pela descoloração da solução complexada pelo reagente 1,5-difenilcarbazida, medida por espectroscopia UV-visível, ${ }^{34}$ no comprimento de onda de $543 \mathrm{~nm}$, utilizando UV 2550, Shimadzu Corparation. Neste método, alíquotas de $0,1 \mathrm{~mL}$ da solução de $\mathrm{Cr}$ (VI) em diferentes tempos de reação foram coletadas e transferidas para um balão volumétrico de $25,00 \mathrm{~mL}$ e adicionados $0,5 \mathrm{~mL}$ do reagente 1,5-difenilcarbazida ( $5 \mathrm{~g} \mathrm{~L}^{-1} \mathrm{em}$ acetona), completando posteriormente o volume do balão com uma solução ácida de $\mathrm{H}_{2} \mathrm{SO}_{4}(\mathrm{pH} 1)$.

\section{RESULTADOS E DISCUSSÃO}

\section{Redução à temperatura programada (TPR)}

A Figura 1 apresenta o resultado da análise TPR para o pó de aciaria elétrica (PAE).

Pode-se notar que o perfil de TPR do PAE apresenta três picos de consumo de hidrogênio em aproximadamente 540,727 e $834^{\circ} \mathrm{C}$,

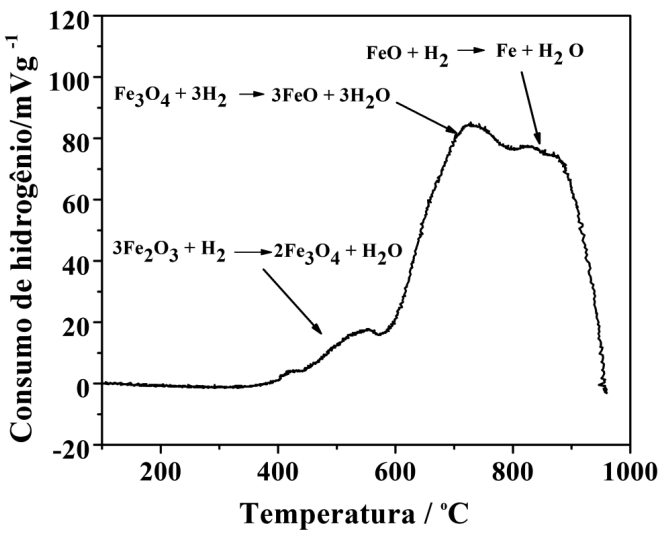

Figura 1. Perfil de redução à temperatura programada (TPR) para o PAE que são atribuídos à redução das fases hematita $\left(\mathrm{Fe}_{2} \mathrm{O}_{3}\right)$, magnetita $\left(\mathrm{Fe}_{3} \mathrm{O}_{4}\right)$ e wustita $(\mathrm{FeO}),{ }^{17,35}$ respectivamente. Estes valores são ligeiramente superiores aos encontrados na literatura, o que indica que as fases de ferro podem estar protegidas por encapsulamento, exigindo maior temperatura para reação com $\mathrm{H}_{2}$. Esta camada protetora pode ser formada pela presença de óxidos mais refratários, como $\mathrm{CaO}, \mathrm{MnO}, \mathrm{SiO}$ e $\mathrm{Al}_{2} \mathrm{O}_{3}$ ou carvão (formado no alto forno durante a produção do aço). ${ }^{17}$ Este resultado foi utilizado como parâmetro para determinar as temperaturas 400,600 e $800^{\circ} \mathrm{C}$ para a realização do tratamento térmico do PAE utilizando gás hidrogênio ou carvão vegetal para obtenção dos compósitos contendo fases reduzidas de ferro.

\section{Difração de raio-X (DRX)}

A Figura 2 apresenta os resultados de DRX do pó de aciaria elétrica e dos materiais obtidos após o tratamento térmico.

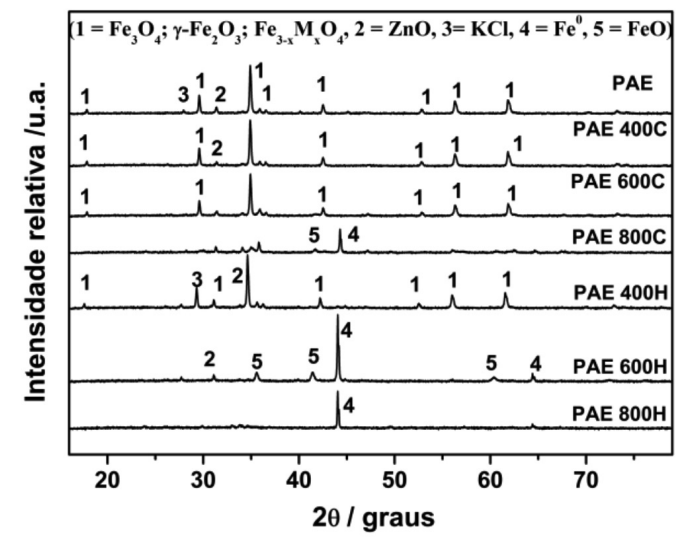

Figura 2. Difratogramas obtidos para o PAE tratado em diferentes temperaturas

Observando o difratograma do PAE, nota-se linhas de difração correspondentes à fase espinélio $\left(\mathrm{Fe}_{3} \mathrm{O}_{4}\right), \mathrm{ZnO}$ e $\mathrm{KCl}$. Conforme resultados obtidos pela análise química o PAE possui quantidades significativas dos metais $\mathrm{Zn}^{2+}(12 \% \mathrm{~m} / \mathrm{m}), \mathrm{Mn}^{2+}(3,2 \% \mathrm{~m} / \mathrm{m})$ e Ca ${ }^{2+}$ $(4,6 \% \mathrm{~m} / \mathrm{m})$, que podem substituir isomorficamente átomos de ferro na magnetita formando $\mathrm{Fe}_{3-\mathrm{x}} \mathrm{M}_{\mathrm{x}} \mathrm{O}_{4}$ (onde $\mathrm{M}=\mathrm{Zn}^{2+}, \mathrm{Mn}^{2+}$ ou $\left.\mathrm{Ca}^{2+}\right){ }^{2,8,35,36}$ Como estas ferritas apresentam linhas de difração coincidentes com os valores de $2 \theta$ da magnetita pura $\left(\mathrm{Fe}_{3} \mathrm{O}_{4}\right)$ e maghemita $\left(\gamma-\mathrm{Fe}_{2} \mathrm{O}_{3}\right)$, estes resultados indicam a presença destas fases no PAE. Outro fator que indica a presença de magnetitas substituídas por zinco e manganês é o parâmetro de rede $\left(\mathrm{a}_{0}\right)$ obtido para a linha de difração mais intensa $\left(35,5^{\circ}\right)$ da fase espinélio. O parâmetro de rede calculado para a fase espinélio presente no PAE é igual a $0,8429 \mathrm{~nm}$ e o de uma magnetita pura $0,8396 \mathrm{~nm} \cdot{ }^{37}$ Quando se compara o valor de $\mathrm{a}_{0}$ obtido para a fase espinélio com o de magnetitas substituídas com zinco ou manganês $\left(\mathrm{Zn}_{\mathrm{x}} \mathrm{Fe}_{3-\mathrm{x}} \mathrm{O}_{4}, \mathrm{a}_{0}=0,8441 \mathrm{~nm}\right.$ e $\mathrm{Mn}_{\mathrm{x}} \mathrm{Fe}_{3-\mathrm{x}} \mathrm{O}_{4}$ $\left.\mathrm{a}_{0}=0,8431\right),{ }^{37-39}$ nota-se que estes estão muito próximos, indicando a presença destas ferritas no PAE.

Os difratogramas obtidos para as amostras PAE400C, PAE600C e PAE400H são semelhantes ao do PAE, indicando que não houve redução significativa das fases de ferro. Por outro lado, nos difratogramas das amostras PAE600H e PAE800C pode-se notar linhas de difração centradas em aproximadamente 42,0 e $44,2^{\circ}$, indicando a presença de wustita $(\mathrm{FeO})$ e ferro metálico, respectivamente, enquanto que o difratograma do PAE800H apresentou linhas de difração referentes à fase $\mathrm{Fe}^{0}$. Estes resultados mostram que o tratamento térmico realizado com $\mathrm{H}_{2}$ foi mais eficiente para promover a redução das fases oxidadas de ferro em relação ao carvão vegetal. 


\section{Microscopia eletrônica de varredura}

A Figura 3 apresenta as micrografias obtidas para o PAE, PAE $800 \mathrm{H}$ e PAE800C.

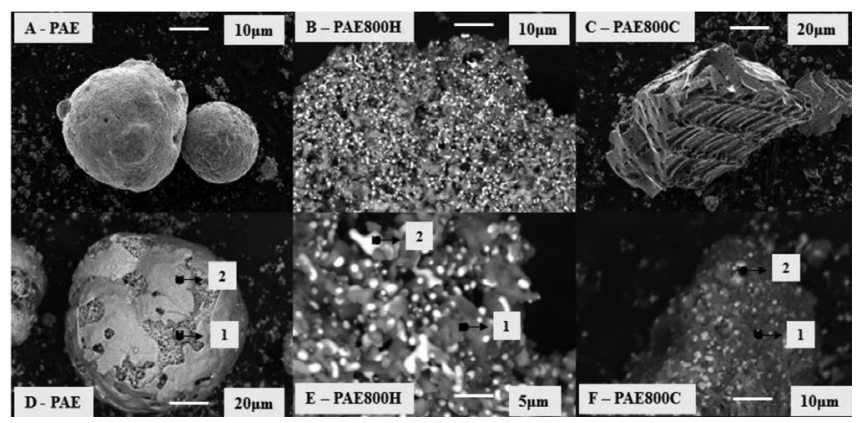

Figura 3. Micrografias obtidas para o PAE, PAE800H e PAE800C - pontos 1 e 2 utilizados para obtenção dos espectros de energia dispersiva

Na micrografia do PAE (Figura 3A) pode-se observar partículas de diferentes tamanhos e formas, sendo a esférica predominante. $\mathrm{O}$ formato esférico do PAE também foi observado em outros trabalhos da literatura e está relacionado ao mecanismo de sua formação, que ocorre pela ejeção de partículas do metal líquido e escória. ${ }^{36,40} \mathrm{Na}$ micrografia do PAE800H (Figura 3B) pode-se notar um aglomerado de partículas onde se distingue pontos de coloração mais clara, o que não foi observado para o PAE. Na microscopia do PAE800C (Figura 3C) nota-se claramente a presença de partícula de carvão, onde pode-se observar a presença de poros em sua superfície. Nota-se também a presença de partículas menores de PAE que aparecem próximas à partícula de carvão como também em sua superfície.

As imagens "D", "E" e "F" apresentadas na Figura 3 apresentam os pontos 1 e 2 das micrografias onde foram obtidos os espectros de EDS para o PAE, PAE800H e PAE800C, respectivamente. Nota-se que os pontos selecionados para obtenção dos espectros EDS possuem tonalidades clara e escura.

A Tabela 1 apresenta a composição química do PAE, PAE800H e PAE800C estimada por EDS para os pontos 1 e 2 das micrografias apresentadas na Figura 3 (D, E e F).

Estes resultados mostram que a composição química nos pontos

Tabela 1. Composição química do PAE, PAE800H e PAE800C nos pontos 1 e 2 - valores estimados pela análise EDS

\begin{tabular}{lcccccc}
\hline & \multicolumn{2}{c}{ PAE } & \multicolumn{2}{c}{ PAE800H } & \multicolumn{2}{c}{ PAE800C } \\
\cline { 2 - 7 } & P1 (\%) & P2 (\%) & P1 (\%) & P2 (\%) & P1 (\%) & P2 (\%) \\
\hline $\mathrm{C}$ & 3 & 3,5 & 5,2 & 3,9 & 24 & 12,8 \\
$\mathrm{O}$ & 9.2 & 8,0 & 17,8 & - & 14 & 2,2 \\
$\mathrm{Mg}$ & 2,0 & - & 9,8 & 0,4 & 9 & 3,7 \\
$\mathrm{Al}$ & 0.5 & - & 1,0 & - & 1,9 & 0,4 \\
$\mathrm{Si}$ & 4 & 2,0 & 17,0 & 0,5 & 2,9 & 1,5 \\
$\mathrm{P}$ & 0.4 & - & - & - & 0,4 & 0,4 \\
$\mathrm{Ca}$ & 6.0 & - & 25,8 & 1,4 & 26,8 & - \\
$\mathrm{Cr}$ & 0.3 & - & - & - & 0,2 & 0,2 \\
$\mathrm{Mn}$ & 4.2 & - & 10,2 & 0,9 & & - \\
$\mathrm{Fe}$ & 61.5 & 85,0 & 12,9 & 92,9 & 20,8 & 75,7 \\
$\mathrm{Zn}$ & 8,9 & 1,5 & - & - & - & - \\
$\mathrm{Na}$ & - & - & 0,3 & - & - & - \\
$\mathrm{Ca}$ & - & - & - & - & - & 3,0 \\
\hline
\end{tabular}

1 e 2 são bastante distintas, onde $\mathrm{P} 1$ possui os seguintes elementos: $\mathrm{C}, \mathrm{O}, \mathrm{Mg}, \mathrm{Al}, \mathrm{Si}, \mathrm{P}, \mathrm{Ca}, \mathrm{Cr}, \mathrm{Mn}, \mathrm{Fe}$ e Zn. Por outro lado a composição do ponto 2 é: $\mathrm{C}, \mathrm{O}, \mathrm{Si}, \mathrm{Fe}$ e $\mathrm{Zn}$, sendo o ferro o elemento majoritário. Ressalta-se que a presença destes elementos no PAE está de acordo com outros trabalhos encontrados na literatura. ${ }^{35,36}$ A presença de maiores teores de ferro no ponto 2 em relação ao ponto 1 para os materiais tratados termicamente, indica a formação de ferro metálico (pontos claros nas imagens) e a presença de zinco, cálcio e manganês indica a presença das fases espinélio $\mathrm{Fe}_{3-\mathrm{x}} \mathrm{Zn}_{\mathrm{x}} \mathrm{O}_{4}, \mathrm{Fe}_{3-\mathrm{x}} \mathrm{Ca}_{\mathrm{x}} \mathrm{O}_{4} \mathrm{e}$ $\mathrm{Fe}_{3-\mathrm{x}} \mathrm{Mn}_{\mathrm{x}} \mathrm{O}_{4}$ no PAE. Resultados semelhantes foram obtidos no trabalho de Machado et al (2004). ${ }^{41}$ É interessante ressaltar que os materiais obtidos a $800{ }^{\circ} \mathrm{C}$ não possuem quantidades significativas de zinco nos pontos 1 e 2 , o que indica que este metal provavelmente evaporou durante o tratamento térmico (Equação 5). A formação de um material de aparência metálica na região fria do reator para as reações realizadas a $800{ }^{\circ} \mathrm{C}$ corroboram estes resultados.

$$
\mathrm{ZnFe}_{2} \mathrm{O}_{4(\mathrm{~s})}+4 \mathrm{C}_{(\mathrm{s})} / 4 \mathrm{H}_{2(\mathrm{~g})} \rightarrow 2 \mathrm{Fe}_{(\mathrm{s})}+\mathrm{Zn}_{(\mathrm{g})}+4 \mathrm{CO}_{(\mathrm{g})} / 4 \mathrm{H}_{2} \mathrm{O}_{(\mathrm{g})}
$$

\section{Área superficial BET}

A Tabela 2 apresenta os valores de área superficial BET obtidos para os materiais estudados.

Tabela 2. Valores de área superficial BET obtidos para o PAE tratado ou não termicamente na presença de $\mathrm{H}_{2}$ (PAE-H) ou carvão (PAE-C)

\begin{tabular}{lccc}
\hline \multirow{2}{*}{ Temperatura $/{ }^{\circ} \mathrm{C}$} & \multicolumn{3}{c}{ Área superficial $/ \mathrm{m}^{2} \mathrm{~g}^{-1}$} \\
\cline { 2 - 4 } & PAE-H & PAE-C & PAE \\
\hline 400 & 3 & 7 & 4 \\
600 & 9 & 36 & \\
800 & 64 & 158 & \\
\hline
\end{tabular}

Estes resultados mostram que a área superficial do PAE $\left(4 \mathrm{~m}^{2} \mathrm{~g}^{-1}\right)$ aumentou significativamente quando este foi reduzido a temperaturas superiores a $600{ }^{\circ} \mathrm{C}$, onde o PAE600C e PAE800C apresentaram aumentos de 9 e 39 vezes, respectivamente, em relação a área do PAE não reduzido. Este aumento pode estar relacionado com a ativação do carvão que ficou impregnado na superfície destes materiais após tratamento térmico. A ativação do carvão vegetal pode ter ocorrido a partir da reação deste com o $\mathrm{CO}_{2}$ formado durante a redução do PAE ou pela reação entre o carvão e os óxidos de ferro. ${ }^{14} \mathrm{O}$ aumento de área superficial observado para o PAE800H possivelmente está relacionada com a volatização do zinco durante o tratamento térmico. A consequência deste processo é a modificação da microestrutura da ferrita, ocasionando na formação de poros devido à vacância na estrutura cristalina. ${ }^{42} \mathrm{~A}$ formação do ferro metálico a partir destes materiais podem gerar imperfeições superficiais no metal, ocasionando o aumento da área superficial.

\section{Reação para redução de cromo (VI)}

Os resultados obtidos nas reações de redução do $\mathrm{Cr}$ (VI) utilizando-se o PAE antes e após tratamento térmico são apresentados na Figura 4.

Estes resultados mostram que o PAE antes da redução apresentou uma considerável atividade, chegando a reduzir ca. $62 \%$ do $\mathrm{Cr}(\mathrm{VI}) \mathrm{em}$ 60 minutos de reação (Figura 4C). Esta atividade apresentada pelo PAE certamente está relacionada à redução do $\mathrm{Cr}(\mathrm{VI})$ pelo $\mathrm{Fe}^{2+}$ presente na fase espinélio, ${ }^{17}$ conforme equação química simplificada (Equação 6):

$$
\left[\mathrm{Fe}^{2+} \mathrm{Fe}_{2}{ }^{3+} \mathrm{O}_{4}\right]+\mathrm{Cr}^{6+} \rightarrow\left[\mathrm{Fe}_{1-\mathrm{x}}{ }^{2+} \mathrm{Fe}_{2+\mathrm{x}}{ }^{3+} \mathrm{O}_{4}\right]+\mathrm{Cr}^{3+}
$$



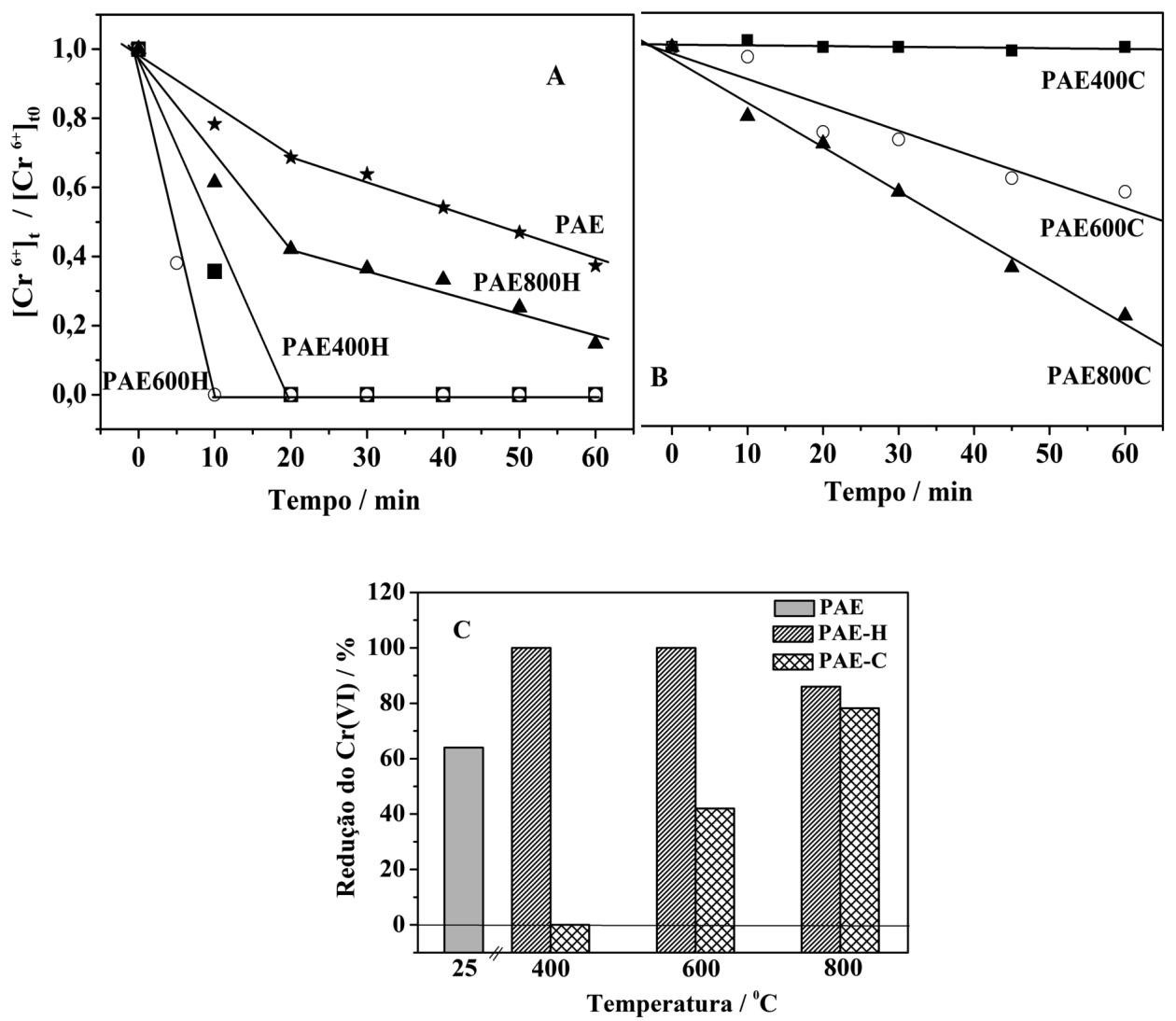

Figura 4. Redução de Cr (VI) $50 \mathrm{mg} \mathrm{L}^{-1}$ utilizando o PAE tratado termicamente com $\mathrm{H}_{2}(\mathrm{~A})$ ou carvão vegetal (B) e percentagem de redução (C)

Os materiais obtidos após tratamento térmico com $\mathrm{H}_{2}$ mostraram atividades bastante elevadas, chegando a reduzir de $85 \%$ a $100 \%$ da concentração inicial de $\mathrm{Cr}(\mathrm{VI})$ até 60 minutos de reação. Ressalta-se que o PAE600H reduziu $100 \%$ do $\mathrm{Cr}(\mathrm{VI})$ em apenas 10 minutos de reação enquanto que o PAE400 demorou cerca de 20 minutos para alcançar a mesma eficiência. A maior eficiência apresentada pelo PAE600H provavelmente está relacionada com a presença das fases $\mathrm{Fe}^{0}$ e $\mathrm{FeO}$. Outros trabalhos da literatura sugerem que materiais contendo as fases $\mathrm{Fe}, \mathrm{FeO}$ e/ou $\mathrm{Fe}_{3} \mathrm{O}_{4}$ possuem um efeito sinérgico capaz de aumentar a eficiência em reações que envolvem transferência de elétrons para outras espécies químicas, como $\mathrm{Cr}(\mathrm{VI})$ e $\mathrm{H}_{2} \mathrm{O}_{2} \cdot{ }^{13,14,16,17,33}$ Apesar do PAE800H possuir maior área superficial e ferro metálico em sua composição, este apresentou menor atividade para a redução do $\mathrm{Cr}(\mathrm{VI})$. Esta menor atividade pode estar relacionada com uma possível oxidação do ferro metálico superficial pela ação do oxigênio atmosférico, ${ }^{16}$ porém são necessárias caracterizações mais detalhadas do material para confirmação.

Na Figura 4B pode-se observar que o PAE400C não apresentou atividade significativa para a redução do $\mathrm{Cr}(\mathrm{VI})$, o que pode estar relacionado com o recobrimento de sua superfície pelo carvão remanescente após tratamento térmico. Por outro lado, os compósitos PAE600C e PAE800C apresentaram atividade para a redução do cromo hexavalente, onde este último foi mais eficiente, chegando a reduzir ca. $78 \%$ em 60 minutos de reação, enquanto que o PAE600C reduziu ca. $42 \%$ (Figura 4C). Esta melhor atividade apresentada pelo PAE800C está relacionada com a presença das fases $\mathrm{Fe}, \mathrm{FeO} \mathrm{e} \mathrm{Fe}_{3} \mathrm{O}_{4}$, conforme observado pelo DRX.

Os resultados apresentados na Figura 4A mostram que a cinética de redução do $\mathrm{Cr}(\mathrm{VI})$ não é simples, porém pode-se determinar duas regiões onde há uma linearidade entre os resultados ( 0 a 20 minutos e 20 a 60 minutos). Nestas regiões foi considerada uma cinética de pseudo-ordem zero e os valores das constantes de velocidade de redução do $\mathrm{Cr}(\mathrm{VI})\left(k_{\text {red }}\right)$ foram obtidas pelos coeficientes angulares das retas que se encontram no intervalo de 0 a 10 ou 20 minutos de reação. Estes resultados são apresentados na Figura 5.

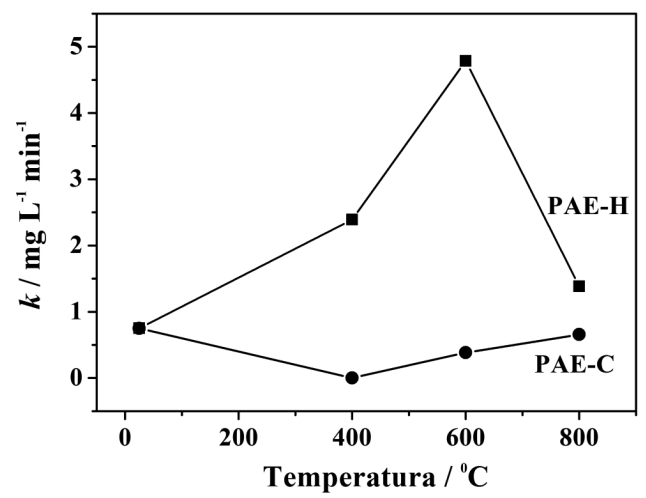

Figura 5. Constante de velocidade de redução do Cr (VI) apresentada pelos materiais obtidos a partir do tratamento térmico do PAE

Estes resultados mostram que o PAE400H e PAE600H apresentaram maior velocidade e eficiência na reação para redução do $\mathrm{Cr}(\mathrm{VI})$, onde os valores de $k_{\text {red }}$ foram ca. 3 e 6 vezes maiores do que a constante de velocidade de redução apresentada pelo PAE, respectivamente. Por outro lado, os materiais obtidos pelo tratamento térmico realizado com carvão vegetal não apresentaram aumento significativo na velocidade das reações.

Devido à possibilidade de ocorrer adsorção do cromo hexavalente pelos materiais estudados, foram realizados testes de dessorção utilizando-se solução de fosfato (Equação 7). ${ }^{14-16}$

$$
\mathrm{CrO}_{4}{ }^{2-}{ }_{\text {(adsorvido) }}+\mathrm{PO}_{4}{ }^{3-}{ }_{\text {(aq) }} \rightarrow \mathrm{CrO}_{4}{ }^{2-}{ }_{\text {(aq) }}+\mathrm{PO}_{4}^{3-}{ }_{\text {(adsorvido) }}
$$


Os resultados obtidos para os testes de dessorção do $\mathrm{Cr}(\mathrm{VI})$ da superfície dos materiais estudados mostraram que os mesmos não adsorveram significativamente o metal. Estes resultados indicam que os materiais estudados, principalmente o PAE400H e PAE600H, possuem grande eficiência e aplicabilidade em reações para redução do $\mathrm{Cr}(\mathrm{VI})$ presente em solução aquosa.

A desativação do PAE600H foi investigada reutilizando o material em várias reações para redução do $\mathrm{Cr}(\mathrm{VI})$ (Figura 6).
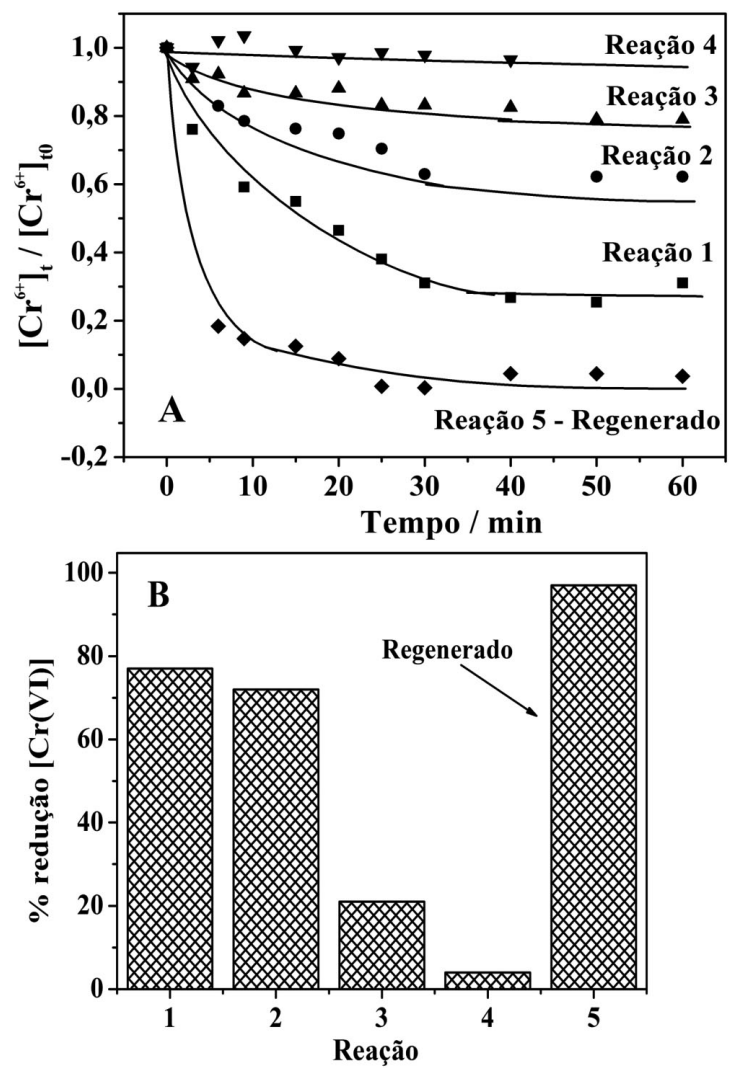

Figura 6. Reutilização do PAE600 para redução do Cr(VI) até sua desativação

Estes resultados mostram que a atividade do PAE600H diminui sucessivamente até próximo a sua completa desativação, que ocorre após a terceira reação. Este efeito é claramente observado na Figura $6 \mathrm{~B}$, onde se pode notar que na primeira reação o PAE600H reduz cerca de $77 \%$ do $\mathrm{Cr}(\mathrm{VI})$ e posteriormente sua eficiência diminui a $5 \%$.

Esta redução de atividade está relacionada com a oxidação das fases reduzidas de ferro ( $\mathrm{Fe}$ e $\mathrm{FeO}$ ) presente no material, levando na formação de fases oxidadas $\left(\mathrm{Fe}^{3+}\right)$, não ativas para a redução do Cr(VI). Esta oxidação foi confirmada após a realização de análises por espectroscopia Mössbauer para o PAE600H antes e após desativação (reação 4). Este resultado é apresentado na Figura 7 e os parâmetros hiperfinos na Tabela 3.

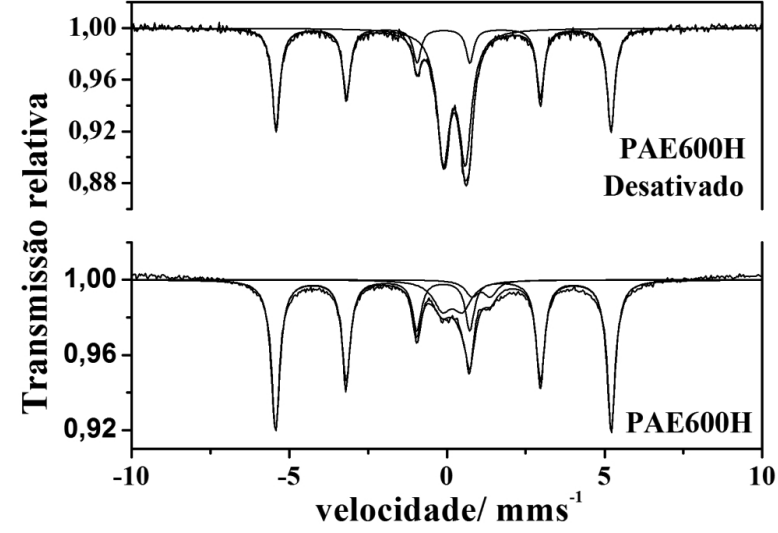

Figura 7. Espectros Mössbauer obtidos para PAE600H antes e após desativação (reação 4)

O espectro Mössbauer obtido para o PAE600H confirma as fases identificadas por DRX, onde se pode observar um sexteto e um dupleto com áreas relativas de 79,1 e 6,0\% referentes ao ferro metálico e à wustita $(\mathrm{FeO})$, respectivamente. $\mathrm{O}$ dupleto superparamagnético com deslocamento isomérico $(\delta) 0,285 \mathrm{mms}^{-1}$ e desdobramento quadrupolar $(\Delta) 0,64 \mathrm{mms}^{-1}$ indicam a presença de óxido de ferro oxidado altamente disperso no PAE600H. No espectro Mössbauer obtido para o PAE600H após desativação, observa-se que a área relativa à fase de $\mathrm{Fe}^{0}$ diminuiu para $50,1 \%$ enquanto que a do $\mathrm{Fe}^{3+}$ aumentou para $49,9 \%$, indicando que as fases reduzidas $\mathrm{Fe}^{0}$ e $\mathrm{FeO}$ sofreram oxidação durante as reações para conversão do $\mathrm{Cr}(\mathrm{VI})$ a Cr(III). A Tabela 3 apresenta os parâmetros hiperfinos obtidos para os espectros Mössbauer, o que confirma a presença destas fases no PAE600H antes e após desativação.

Após a desativação do PAE600H, este foi reativado pelo tratamento térmico a $600{ }^{\circ} \mathrm{C}$ durante 30 minutos sob atmosfera de $\mathrm{H}_{2}(100$ $\left.\mathrm{ml} \mathrm{min}{ }^{-1}\right)$. O resultado desta reação mostra que o PAE600H reativado reduziu $96 \%$ da concentração inicial de $\mathrm{Cr}(\mathrm{VI})$. Esta elevada eficiência é devido à redução de fases oxidadas presentes no PAE600H desativado, ${ }^{14,15}$ comprovando que a reativação sob fluxo de $\mathrm{H}_{2}$ foi eficiente.

\section{CONCLUSÃO}

Os resultados obtidos neste trabalho mostraram que o tratamento térmico do PAE na presença de hidrogênio ou carvão vegetal promove a redução de fases oxidadas de ferro formando $\mathrm{Fe}^{0}, \mathrm{FeO}$ e $\mathrm{Fe}_{3} \mathrm{O}_{4}$. No entanto, o tratamento térmico sob atmosfera de hidrogênio foi mais eficiente que sob presença de carvão vegetal, no qual o material obtido na temperatura de $600^{\circ} \mathrm{C}(\mathrm{PAE} 600 \mathrm{H})$ apresentou maior eficiência na redução de cromo hexavalente apresentando uma constante de velocidade de redução de cromo de $4,8 \mathrm{mg} \mathrm{L}^{-1} \mathrm{~min}^{-1}$.

Os materiais obtidos podem ser facilmente reativados após perda de atividade, pelo tratamento térmico na presença de agente redutor.

Tabela 3. Parâmetros hiperfinos Mössbauer para PAE600H antes e após desativação

\begin{tabular}{|c|c|c|c|c|c|}
\hline Amostra & Sítio ${ }^{57} \mathrm{Fe}$ & $\delta / \mathrm{mm} \mathrm{s}^{-1}$ & $\varepsilon, \Delta / \mathrm{mm} \mathrm{s}^{-1}$ & $B_{\mathrm{hf}} / \mathrm{T}$ & $A R / \%$ \\
\hline \multirow[t]{3}{*}{ PAE600H } & $\mathrm{Fe}^{0}$ & $-0,0038$ & 0,00 & 32,98 & 79,1 \\
\hline & Wt & 1,196 & 0,59 & ----- & 6,0 \\
\hline & $\mathrm{Fe}^{3+}$ & 0,285 & 0,64 & ----- & 14,9 \\
\hline \multirow[t]{2}{*}{ PAE600H Desativado } & $\mathrm{Fe}^{0}$ & $-0,002$ & 0,00 & 32,91 & 50,1 \\
\hline & $\mathrm{Fe}^{3+}$ & 0,347 & 0,69 & ----- & 49,9 \\
\hline
\end{tabular}

$\delta=$ deslocamento isomérico relativo ao $\alpha \mathrm{Fe} ; \varepsilon=$ deslocamento quadrupolar; $\Delta=$ desdobramento quadrupolar; $B_{\mathrm{hf}}$ = campo magnético hiperfino; $A R=$ área subespectral relativa. $\mathrm{Wt}=$ wustita e $\mathrm{Fe}^{0}=$ ferro metálico. 
Diante do exposto, nota-se que é possível agregar valor ao resíduo PAE através de sua redução térmica, o que potencializa sua aplicabilidade em reações para remediação ambiental.

\section{AGRADECIMENTOS}

À FAPEMIG, CAPES e CNPQ pelo apoio financeiro. Ao Centro de Microscopia da Universidade Federal de Minas Gerais (http:// www.microscopia.ufmg.br) pelos equipamentos e suporte técnico nos experimentos envolvendo microscopia.

\section{REFERÊNCIAS}

1. Machado, J. G. M. S.; Brehm, F. A.; Moraes, C. A. M.; Santos, C. A.; Vilela, A. C. F.; J. Mater. Res. 2006, 9, 41.

2. Menad, N.; Ayala, J. N.; Garcia-Carcedo, F.; Ruiz-Ayucar, E.; Hernandez, A.; Waste Manage. 2003, 23, 483.

3. Souza, C. A. C. S.; Machado, A. T.; Lima, L. R. P. A.; Cardoso, R. J. C. C.; J. Mater. Res. 2010, 13, 513.

4. NBR, 2004. Norma Brasileira 10004: Resíduos sólidos - Classificação. ABNT.

5. Machado, G. M. S. J.; Brehm, A. F.; Moraes, M. A. C.; Santos, A. C.; Vilela, F. C. A.; Cunha, M. B. J.; J. Hazard. Mater. B 2006, 136, 953.

6. Lenz, D. M.; Martins, F. B.; Revista Matéria 2007, 12, 503.

7. Tsubouchi, N.; Hashimoto, H.; Ohtaka, N.; Ohtsuka, Y.; J. Hazard. Mater. 2010, 183, 116.

8. Maslehuddin, M.; Awan, F. R.; Shameem, M.; Ibrahim, M.; Ali, M. R.; Constr. Build. Mater. 2011, 25, 308.

9. Salihoglu, G.; Pinarli, V.; J. Hazard. Mater. 2008, 153, 1110.

10. Hatim, I.; Marija, K.; Milorad, G.; Revista de Chimie 2012, 63, 1008

11. Lis, T.; Nowacki, K.; Steel Res. Int. 2012, 83, 842.

12. Mecozzi, R.; Palma, L., DI.; Pilone, D.; Cerboni, L.; J. Hazard. Mater. 2006, 137, 886 .

13. Costa, R. C. C.; Moura, F. C. C.; Oliveira, P. E. F.; Magalhães, F.; Ardisson, J. D.; Lago, R. M.; Chemosphere 2010, 78, 1116.

14. Magalhães, F.; Pereira, M. C.; Fabris, J. D.; Bottrel, S. E. C.; Sansiviero, M. T. C.; Amaya, A.; Tancredi, N.; Lago, R. M.; J. Hazard. Mater. 2009, $165,1016$.

15. Pereira, M. C.; Coelho, F. S.; Nascentes, C. C.; Fabris, J. D.; Araújo, M. H.; Sapag, K.; Oliveira, L. C. A.; Lago, R. M.; Chemosphere 2010, 81, 7.

16. Oliveira, P. E. F.; Oliveira, L.; Ardisson, J. D.; Lago, R. M.; J. Hazard. Mater. 2011, 194, 393.
17. Amorim, C. C.; Dutra, P. R.; Leão, M. M. D.; Pereira, M. C.; Henriques, A. B.; Fabris, J. D.; Lago, R. M.; Chem. Eng. J. 2012, 209, 645.

18. Xia, S.; Zhang, W.; Chen, L.; Chen, H.; J. Hazard. Mater. 2007, 143, 57.

19. Liu, G. H.; Zhu, S. N.; Ye, Z.; Water Air Soil Pollut. 2012, 223, 5049.

20. Xu, J.; Bhattacharyya, D.; J. Phys. Chem. C 2008, 112, 9133.

21. Peralta-Zamora, P.; Cavalotti, L. F. R.; Rodrigues, M. B.; Paiva, T. C.; Quim. Nova 2009, 32, 1504.

22. Tong, M.; Yuan, S.; Long, H.; Zheng, M.; Wang, L.; Chen, J.; J. Contam. Hydrol. 2011, 122, 16.

23. Zhai, G; Xie, L; Luan, F; Sheng, J; Li, J , Zhou, Q.; J. Hazard. Mater. 2012, 217, 416.

24. Vantelon, D.; Mullet, M.; Demoisson, F.; Humbert, B.; Michot, L. J.; Geochim. Cosmochim. Acta. 2007, 71, 3257.

25. Park, J. M.; Park, D.; Yun, Y. S.; J. Colloid Interface Sci. 2008, 317, 54.

26. Deng, B.; Qing, L. Y.; Xiang, Y. L.; Pedosphere 2006, 16, 572.

27. Souza, L. R. C.; Peralta-Zamora, P.; Quim. Nova 2005, 28, 226.

28. Diels, L.; Dries, J.; Bastiaens, L.; Springael, D.; Kuypers, S.; Aghatos, S. N.; Water Res. 2005, 39, 3531.

29. Chang, L. Y.; Environ. Prog. 2005, 24, 305.

30. Allred, B. J.; Ground Water Monit. Rem. 2012, 32-2, 81.

31. Jinlou, H.; Xin, C.; Qingbao, G.; Disaster advances 2012, 5, 105.

32. Mueller, N. C.; Braun, J.; Bruns, J.; Černík, M.; Rissing, R.; Rickerby, D.; Nowack, B.; Environ. Sci. Pollut. Res. 2012, 19, 550.

33. Brehm, F. A.; Graffitti, D. F.; Moraes, C. A. M.; Vilela, A. C. F. Anais do I congresso em ciências dos materiais do MERCOSUL, Joinville, Brasil, 2002.

34. Walsh, A. R.; O’Halloran, J.; Water Res. 1996, 30, 2401.

35. Costa, R. C. C.; Moura, F. C. C.; Ardisson, J. D.; Fabris, J. D.; Lago, R. M.; Appl. Catal., B 2008, 83, 131.

36. Telles, V. B.; Dissertação de Mestrado, Escola Politécnica da Universidade de São Paulo, Brasil, 2010.

37. Galembeck, A.; Gushikem, Y.; Quim. Nova 1996, 19, 200.

38. Baykal, A.; Koseoglu, Y.; Toprak, S. M.; Gozuak, F.; Basaran, C. A.; Aktas, B.; J. Alloys Compd. 2008, 462, 209.

39. Li, Y. H.; Kouh, T.; Shim, I. B.; Kim, C. S.; J. Appl. Phys. 2012, 111, 07 B544.

40. Mantavoni, M. C.; Takano, C.; Buchler, P. M.; Ironmak. Steelmak. 2004, 31,325 .

41. Machado, J. G. M. S.; Dissertação de Mestrado, Universidade Federal do Rio Grande do Sul, Brasil, 2004.

42. Tsugita, Y.; Mater. Trans. 2003, 44, 2422. 